\title{
The mixing of cohesive and flowable powder materials using a common laboratory powder mixer
}

\author{
${ }^{1}$ Mohd Radzuan, N., ${ }^{1, *}$ Anuar, M.S. and ${ }^{2}$ Tahir, S.M. \\ ${ }^{I}$ Department of Process and Food Engineering, Faculty of Engineering, Universiti Putra Malaysia, 43400 \\ UPM Serdang, Selangor, Malaysia. \\ ${ }^{2}$ Department of Mechanical and Manufacturing Engineering, Faculty of Engineering, Universiti Putra \\ Malaysia, 43400 UPM Serdang, Selangor, Malaysia.
}

\section{Article history:}

Received: 10 April 2020

Received in revised form: 22

May 2020

Accepted: 15 July 2020

Available Online: 3 January

2021

Keywords:

Cohesive,

Mixing,

Flowability,

Tumblers,

Homogeneity,

Drum mixers

DOI:

https://doi.org/10.26656/fr.2017.5(S1).004

\begin{abstract}
This study presented the homogeneity obtained when mixing cohesive and flowable powder materials using a laboratory powder mixer. The mixing process parameters studied were the mixing time and the mixer rotational speed (20 rpm, $40 \mathrm{rpm}$ and $60 \mathrm{rpm})$ at the different ratios (95\%: 5\%,50\%: 50\% and 5\%: 95\%) of the cohesive cocoa and flowable mannitol powder materials. The homogeneity sampled at the powder bed surface showed that only at the highest rotational speed of $60 \mathrm{rpm}$ used in this work yield acceptable homogeneity at the two extremes of the powder mass ratios; $95 \%$ : $5 \%$ and $5 \%$ : 95\% of mannitol: cocoa for some of the locations on the powder bed surface, especially near the wall of the mixer. Other combinations of the experimental conditions did not yield acceptable mixture homogeneity. These results showed the difficulties in obtaining a homogeneous powder mix when mixing cohesive powder materials, especially in academic teaching and research laboratories using a simple powder mixer apparatus.
\end{abstract}

\section{Introduction}

Powder mixing is a frequently encountered unit operation in many processes (Asachi and Nourafkan, 2018). Powder mixing is a complex process that is dependent upon the type of equipment used and the raw material to be mix (Bridgwater, 2012). Recently, a number of works attempted to elucidate the complex mechanisms of powder mixing using computer simulations to gain further understanding of the individual particle movements during the mixing process (Gui et al., 2010; Zhang et al., 2017; Zhang et al., 2019). This is to further support the needs of the industry where uniformity of the powder mixture is crucial such as in the pharmaceutical industry (Muzzio et al., 2002). The industrial use of continuous in-line noninvasive techniques to monitor powder mixture uniformity (Gerich et al., 2017) reflects the importance of the mixing stage for commercial production to ensure the final product uniformity is within the acceptable regulatory limits (Cholayudth, 2017). Meanwhile, all of these were seen to be more geared towards the industrial needs, one must not neglect the importance of powder mixture preparation for smaller-scale use such as for academic teaching and research laboratories. These laboratories sometimes were not equipped with sophisticated equipment for powder mixing purposes. In some cases, powder mixing was conducted manually through the use of dilution, manual shaking of containers containing the powder mix or simply hand mixing using a spatula inside a container. In our laboratory, we utilized a laboratory powder rotator for the mixing of the powder materials where these powder mixtures were subsequently compacted into tablet form.

The powder flowability has a large influence on the mixing performance of the powder (Chaudhuri et al., 2006). Cohesive powders are normally difficult to mix and need special equipment for mixing. Therefore, this work is aimed to highlight the difficulties in achieving homogeneity when mixing with a cohesive powder using a common laboratory powder mixer.

\section{Materials and methods}

\subsection{Raw material}

Mannitol and cocoa powders were used in this mixing experiment. The source of the material could not be disclosed in this work due to proprietary reasons. 


\subsection{Raw material physical properties characterization}

\subsubsection{Particle size}

The particle sizes of the cocoa and mannitol powders materials were determined by using a laser light diffraction equipment (Malvern Mastersizer 2000, Malvern, Malvern Instrument Ltd, UK). The median particle size $\left(d_{50}\right)$, was used as the parameter to represent the particle size of the powders used in this work.

\subsubsection{Tapped density}

About $2 \mathrm{~g}$ of each cocoa and mannitol powder samples was weighed and inserted into the $10 \mathrm{~mL}$ graduated cylinder and dropped 120 times on a flat rubber mat from a height of $15 \mathrm{~cm}$. Afterwards, the sample was then inserted into the tap density analyzer (GeoPyc 1360, Micromeritics, Norcross, USA) to obtain the tapped density of the powder sample (Shamjuddin et al., 2014).

\subsubsection{Bulk density}

About $2.5 \mathrm{~g}$ of each cocoa and mannitol powder samples were loaded into $15 \mathrm{~mL}$ graduated cylinder and placed onto a flat and smooth surface. The bulk density was then calculated using by dividing the mass of the powder with the volume of the powder bed (Shamjuddin et al., 2014).

\subsubsection{Flowability assessment}

The flowability of each cocoa and mannitol powder materials was assessed using the simple angle of repose and Carr index values (Carr, 1965; Anuar et al., 2019). These methods are commonly used to characterize the flowability of powders (Shah et al., 2008). For the measurement of the angle of repose, the powder was poured inside a funnel at a fixed height of $10 \mathrm{~cm}$. The powder would then flow through the funnel to form a heap on the flat horizontal surface below the funnel. The angle of the heap was then measured and taken as the angle of repose for the powder. Meanwhile, the Carr index was calculated using the values of the tapped and bulk densities by using:

$$
C I=100 \times \frac{\rho_{t}-\rho_{b}}{\rho_{t}}
$$

Where $C I$ is the Carr index, $\rho_{\mathrm{t}}$ is the tapped density, and $\rho_{\mathrm{b}}$ is the bulk density.

\subsection{Raw material preparation for mixing experiment}

Cocoa and the mannitol powders were mixed in three different mass ratios (Table 1) consisting of $50 \%$ : $50 \%$ mass ratio of the mannitol powder with the cocoa powder, 95\%: 5\% mass ratio of the mannitol powder with the cocoa powder and finally 5\%: $95 \%$ mass ratio of the mannitol powder with the cocoa powder.
Table 1. Raw material preparation.

\begin{tabular}{ccc}
\hline \multirow{2}{*}{ Mass ratio of mixture } & \multicolumn{2}{c}{ Mass of pure material $(\mathrm{g})$} \\
\cline { 2 - 3 } & Mannitol powder & Cocoa powder \\
\hline 50\% mannitol: 50\% cocoa & 100 & 100 \\
95\% mannitol: 5\% cocoa & 190 & 10 \\
$5 \%$ mannitol: $95 \%$ cocoa & 10 & 190 \\
\hline
\end{tabular}

\subsection{Mixing experiment}

The powder samples were then placed into a laboratory powder mixer (Dry Powder Rotator, Glas-Col, Terre Haute, USA) according to the required mass ratios as stated in Table 1. The cylindrical mixing container has a diameter of approximately $8 \mathrm{~cm}$ with a height of $20 \mathrm{~cm}$ and was filled approximately at $30 \%$ capacity. The mixing action of the laboratory powder mixer involved the rotation of the mixing container at an axis thus the powder mixture was tossed from end-to-end inside it. The mannitol powder was inserted first into the mixing container. This was then followed by the insertion of the cocoa powder on top of the mannitol powder. The total mass of the powder mixture in the mixer was $200 \mathrm{~g}$ for each mixing run (Table 1). The mixing process was conducted for a total of 5 minutes mixing time at various constant rotational speeds of 20,40 and $60 \mathrm{rpm}$. The mixing process was interrupted every one minute in order to examine the homogeneity of the mixture. Samples were collected at four sampling positions (Figure 1) within the mixer to ensure a thorough characterization of the homogeneity of the powder mixture. Position 1 represented the sampling position taken in the middle of the mixer. Position 2 and 3 were sampling positions near the wall of the mixer. Meanwhile, position 4 was the area between the middle and wall of the mixer. The samples were drawn at the surface of the powder bed at these positions as depicted in Figure 1. These positions were chosen by considering areas that might have different shear conditions during the mixing process. Three samples having a mass of approximately $1 \mathrm{~g}$ each were withdrawn from each position at one-minute time interval during the 5-minute mixing process.

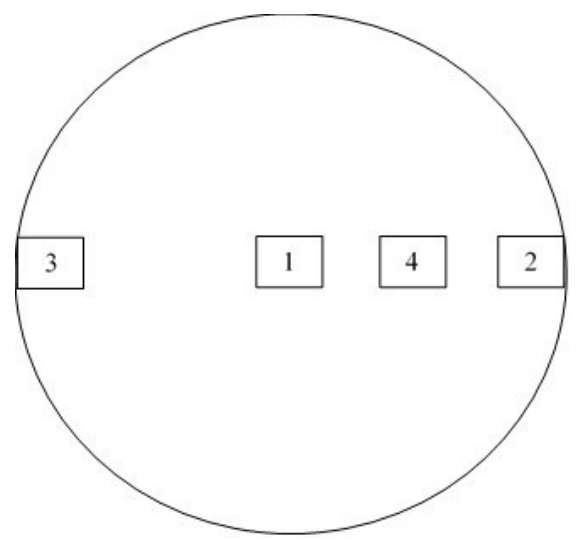

Figure 1.Sampling position in the mixer (top view). 


\subsection{Mixture homogeneity quantitative measurement}

The homogeneity of the powder mixture was determined by analyzing the homogeneity of samples taken at the different positions in the mixer during the mixing process as discussed in Section 2.4 previously. Sample (1 g) taken was then dissolved in a $15 \mathrm{~mL}$ distilled water and stirred for 2 mins. The dissolved mixture was filtrated using a dried filter paper (Permula Chemical, Malaysia). The dried filter paper was weighed before conducting the filtration process. The undissolved sample was trapped on the dry filter paper during this filtration process. Subsequently, the drying of the filter paper containing the undissolved sample was conducted in an oven (Memmert, Germany) at the temperature of $105^{\circ} \mathrm{C}$ for $24 \mathrm{hrs}$. After drying, the dried filter paper along with the dried undissolved sample was weighed. Mass of the undissolved sample was calculated by:

$$
m_{u s}=\left(m_{w f}-m_{d f}\right)
$$

Where $m_{u s}$ is the mass of the undissolved sample after drying, $m_{w f}$ is the total mass of the dried filter with the dried filtered sample on it and $m_{d f}$ is the mass of the dried filter paper prior to the filtration process.

In order to obtain the homogeneity of the samples taken from the mixer, the mass of the undissolved sample would then be compared with the mass of the undissolved sample in a known mass ratio of the mannitol and cocoa powders, which was set as the targeted homogeneity for comparison purposes. For approximately $1 \mathrm{~g}$ of mixing $50 \%$ of mannitol with $50 \%$ cocoa powder, the amount obtained for the targeted homogeneity was $0.570 \mathrm{~g}$ mass of the undissolved sample. Therefore, $0.570 \mathrm{~g}$ of undissolved sample was set as the target specification with an acceptable range of $0.570 \mathrm{~g} \pm 15 \%$. Meanwhile, the target specification with an acceptable range $0.202 \mathrm{~g} \pm 15 \%$ was set for the mixing of $95 \%$ mannitol with 5\% cocoa powder. For the mixing of $5 \%$ mannitol with $95 \%$ cocoa powder, the target specification with an acceptable range is $0.713 \mathrm{~g}$ $\pm 15 \%$ was set. This was a first-order analysis of the homogeneity of the samples, which required no additional chemicals and sophisticated analytical tools.

\section{Results and discussion}

Generally, the cocoa powder used in this work had a smaller median particle size in comparison with the mannitol powder sample as indicated in Table 2. The cocoa powder exhibited very poor flowability characteristics that could be seen through the relatively higher angle of repose and Carr index values (Table 2) compared to the mannitol powder. In contrast, the mannitol powder possessed better flowability characteristics represented by the lower values of the angle of repose and the Carr index compared to cocoa powder as shown in Table 2 . These wide variations between both the powder materials, where the cocoa powder not just having relatively smaller particle sizes but also very cohesive in comparison to the mannitol powder was deemed suitable to be used as a model material to be mixed with the fairly flowable mannitol powder in this study.

Table 1. Raw material preparation.

\begin{tabular}{lcc}
\hline Material properties & Cocoa powder & Mannitol powder \\
\hline Bulk density $\left(\mathrm{kg} / \mathrm{m}^{3}\right)$ & $330.94 \pm 0.03$ & $541.57 \pm 0.04$ \\
Tapped density $\left(\mathrm{kg} / \mathrm{m}^{3}\right)$ & $657.70 \pm 0.003$ & $646.00 \pm 0.002$ \\
Median particle size $(\mu \mathrm{m})$ & $12.27 \pm 0.90$ & $345.89 \pm 0.60$ \\
Angle of repose $\left({ }^{\circ}\right)$ & $44.33 \pm 0.05$ & $36.33 \pm 0.08$ \\
Carr's index & 49.68 & 16.17 \\
Flowability & Extremely poor & Fairly flowable \\
\hline
\end{tabular}

Figures 2-10 illustrate the homogeneity of the cocoamannitol powder mixed at different mass ratios and mixer rotational speeds. It can be clearly observed from Figures 2-10, the best homogeneity that can be achieved was when there was a higher mass of the cohesive cocoa powder $(95 \%)$ compared to the flowable mannitol powder $(5 \%)$ at the highest rotational speed of $60 \mathrm{rpm}$. The homogeneity was achieved within the acceptable range in the initial duration of the mixing for some of the positions on the surface of the powder bed, where towards the end of the mixing process, none of the mixture sampled at the surface of the bed achieved homogeneity within the acceptable limit (Figure 2). Other conditions that achieved the required homogeneity was when the lowest amount of cohesive cocoa powder (5\%) and highest amount of flowable mannitol powder $(95 \%)$ were used also at the highest rotational speed of $60 \mathrm{rpm}$. The similarity for both conditions was that the acceptable homogeneity was observed for samples obtained from the powder bed surface near the wall of the mixer. This finding could be explained based upon aprevious study, where the zone near the wall was found to experience relatively higher particle movements during mixing (Soni et al., 2016) hence this might contribute to the observed homogeneity found for the powder bed surface near the wall. In addition, the use of the highest rotational speeds resulted in the mixture meeting the required homogeneity limit at both mixture compositions. For all other mixture composition and rotational speed combinations, none of the positions sampled on the powder bed surface achieved the acceptable homogeneity (Figures 3-10). Also, based upon the general trend observed, the increase in the mixing time led to a more 'uniform' powder bed, 


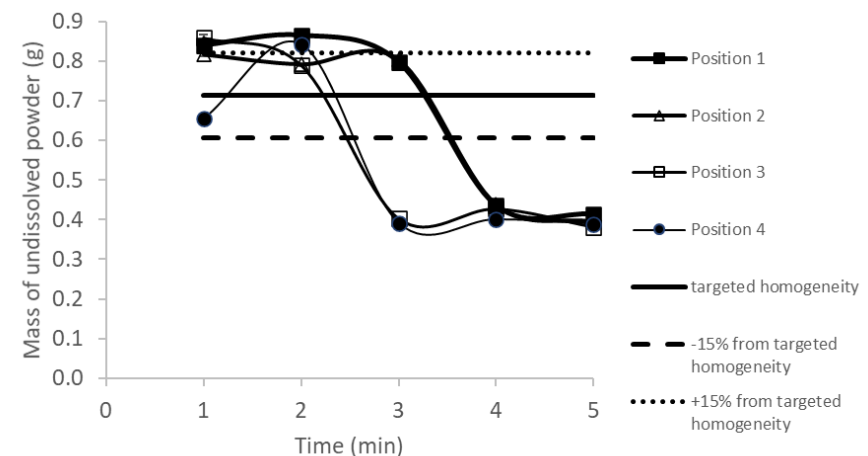

Figure 2 . Homogeneity of 5\% mannitol: $95 \%$ cocoa mixture at $60 \mathrm{rpm}$ mixing speed.

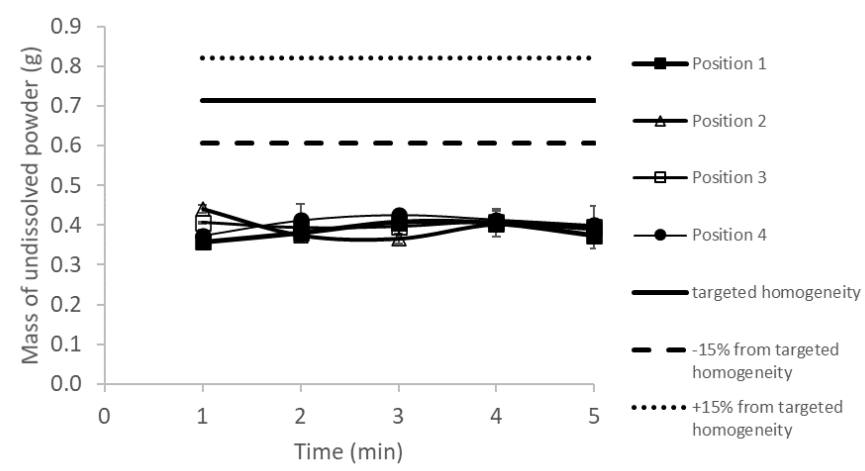

Figure 4. Homogeneity of 5\% mannitol: 95\% cocoa mixture at $20 \mathrm{rpm}$ mixing speed.

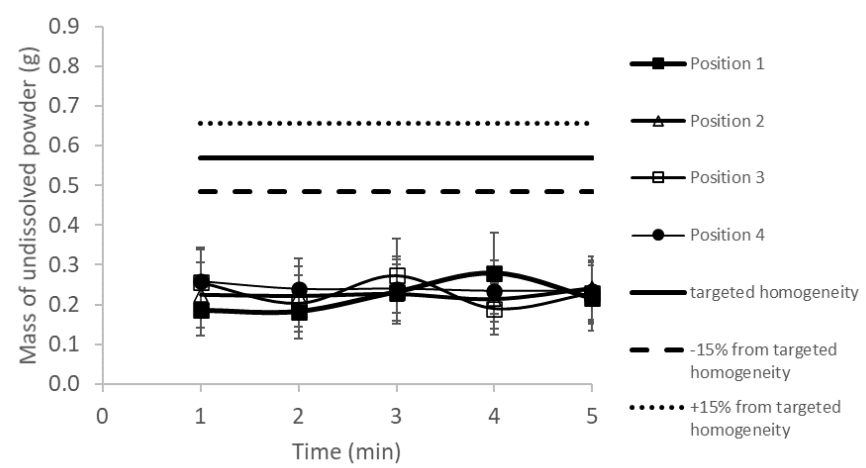

Figure 6 . Homogeneity of $50 \%$ mannitol: $50 \%$ cocoa mixture at $40 \mathrm{rpm}$ mixing speed.

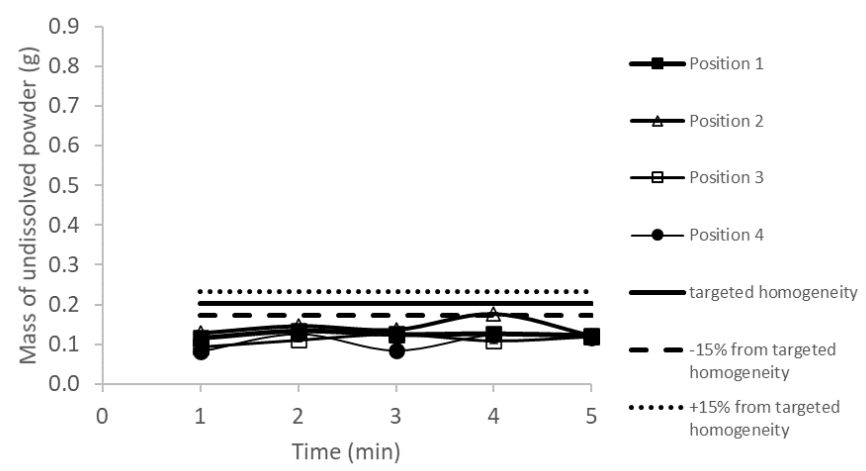

Figure 8 . Homogeneity of $95 \%$ mannitol: $5 \%$ cocoa at $60 \mathrm{rpm}$ mixing speed.

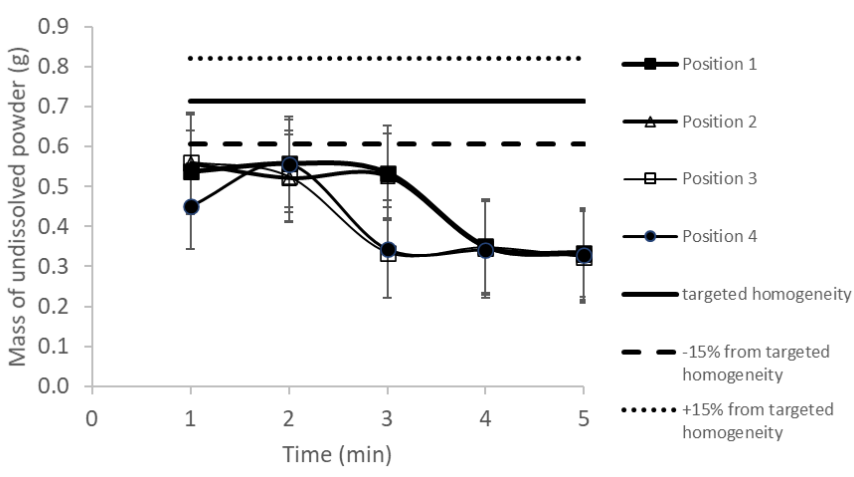

Figure 3. Homogeneity of 5\% mannitol: $95 \%$ cocoa mixture at $40 \mathrm{rpm}$ mixing speed.

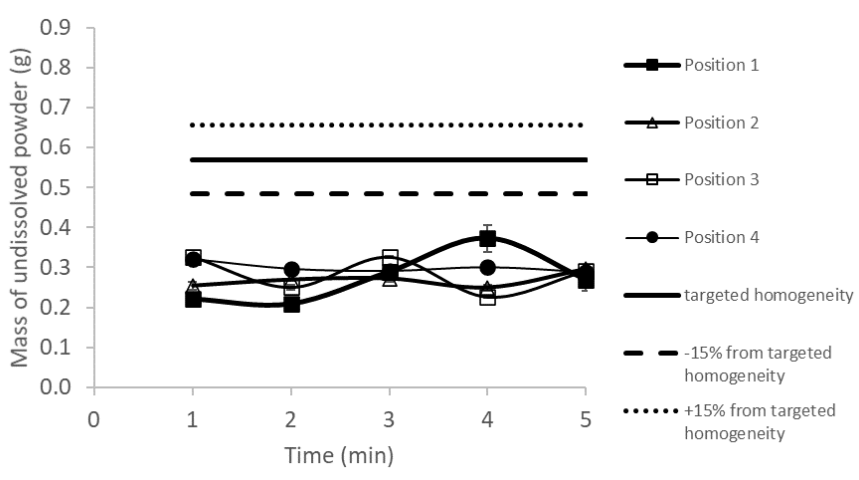

Figure 5. Homogeneity of 50\% mannitol: 50\% cocoa mixture at $60 \mathrm{rpm}$ mixing speed.

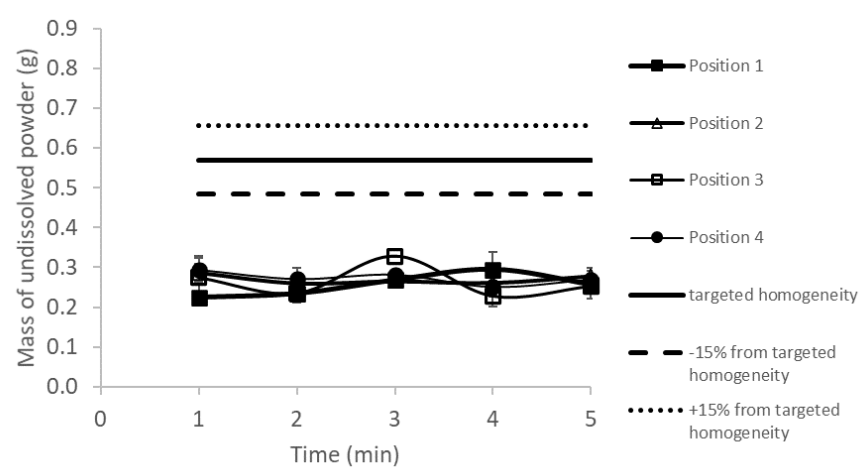

Figure 7. Homogeneity of 50\% mannitol: $50 \%$ cocoa mixture at $20 \mathrm{rpm}$ mixing speed.

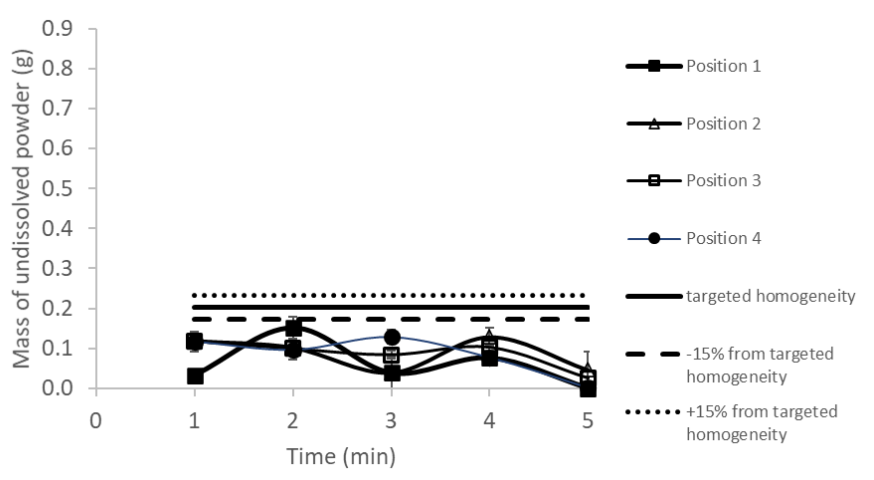

Figure 9 . Homogeneity of $95 \%$ mannitol: $5 \%$ cocoa mixture at $40 \mathrm{rpm}$ mixing speed. 


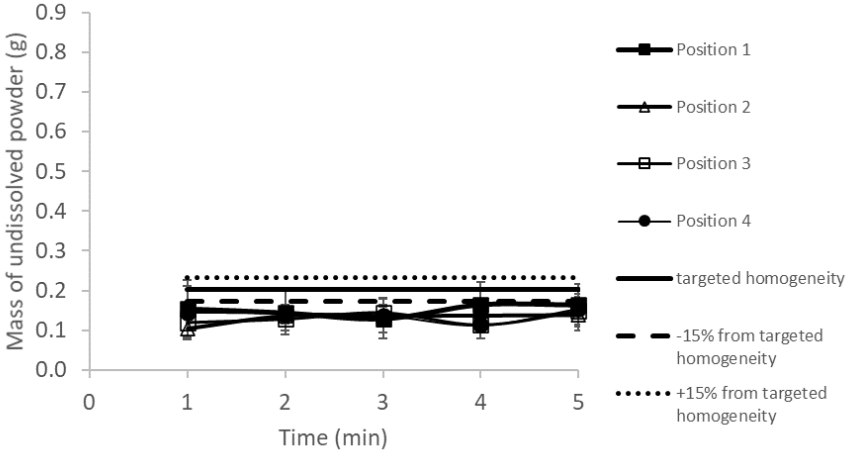

Figure 10 . Homogeneity of $95 \%$ mannitol: $5 \%$ cocoa mixture at $20 \mathrm{rpm}$ mixing speed.

although the homogeneity of the powder bed surface was found to be unacceptable based upon the required homogeneity of the mixture. This was because the undissolved mass of the samples obtained at the different locations on the powder bed surface were becoming more similar as depicted by lower variations between the positions as illustrated in Figures 2-10. Similarly, slowing the rotational speed also led to lesser variations in the homogeneity between the different positions on the powder bed surface, which were located near the wall, middle as well as between the wall and the middle of the powder bed indicating increased uniformity at the powder bed surface.

\section{Conclusion}

Poor mixing performance was achieved during the mixing of the cohesive cocoa and flowable mannitol powders at the rotational speed and composition combinations used in this work. This work showed that the acceptable homogeneity was only achieved at the two extremes of the powder mass ratios; $95 \%$ : $5 \%$ and 5\%: 95\% of mannitol: cocoa for some of the locations on the powder bed surface, especially near the wall of the mixer. This highlight the importance of the proper selection of mixing procedures involving cohesive powders during powder mixture preparation in laboratories especially for academic teaching and research purposes. Preparation of the sample mixture individually to eliminate inhomogeneity due to sampling thus ensuring each sample has the required composition as well as optimizing the mixing parameters before proceeding to subsequent processes such as tabletting can be implemented to ensure the final product achieved the acceptable uniformity.

\section{Acknowledgement}

The authors gratefully acknowledged the funding received from Universiti Putra Malaysia IPS grant vot no: 9660700 and from UPM RMC.

\section{References}

Anuar, M.S., Tahir, Najeeb, M.I. and Ahmad, S. (2019). Banana (Musa acuminate) peel drying and powder characteristics obtained through shade and microwave drying process. Adances in Materials and Processing Technologies, 5(2), 181-190. https:// doi.org/10.1080/2374068X.2018.1545201

Asachi, M. and Nourafkan, E. (2018). A review of the current techniques for the evaluation of powder mixing. Advanced Powder Technology, 29(7), 15251549. https://doi.org/10.1016/j.apt.2018.03.031

Bridgwater, J. (2012). Mixing of powders and granular materials by mechanical means-a perspective. Particuology, 10(4), 397-427. https:// doi.org/10.1016/j.partic.2012.06.002

Carr, R.L. (1965). Evaluating flow properties of solids. Chemical Engineering, 72, 163-169.

Chaudhuri, B., Mehrotra, A., Muzzio, F.J. and Tomassone, M.S. (2006). Cohesive effects in powder mixing in a tumbler blender. Powder Technology, 165(2), 105-114. https://doi.org/10.1016/ j.powtec.2006.04.001

Cholayudth, P. (2017). Establishing blend uniformity acceptance criteria for oral solid-dosage forms. Pharmaceutical Technology, 41(2), 45-52.

Gerich, A., Damen, M., Verhoeven, W., Verhoog, J., Chamarthy, S.P. and Besseling, R. (2017). Detection of lumps in powder blends by inline NIR. Pharmaceutical Technology, 41(2), 36-44.

Gui, N., Fan, J. and Cen, K. (2010). A macroscopic study of particle mixing in a rotating tumbler. Chemical Engineering Science, 65(10), 3034-3041. https:// doi.org/10.1016/j.ces.2010.01.023

Muzzio, F.J., Shinbrot, T. and Glasser, B.J. (2002). Powder technology in the pharmaceutical industry: the need to catch up fast. Powder Technology, 124(1 -2), 1-7. https://doi.org/10.1016/S0032-5910(01) 00482-X

Shah, R.B., Tawakkul, M.A. and Khan, M.A. (2008). Comparative evaluation of flow for pharmaceutical powders and granules. AAPS PharmSciTech, 9, 250258. https://doi.org/10.1208/s12249-008-9046-8

Shamjuddin, A., Anuar, M.S. and Tahir, S.M. (2014). Characteristics of tableted roselle (Hibiscus sabdariffa Linn.) with addition of sodium starch glycolate. Particulate Science and Technology, 32 (4), 384-391. https:// doi.org/10.1080/02726351.2014.880095

Soni, R.K., Mohanty, R., Mohanty, S. and Mishra, B.K. (2016). Numerical analysis of mixing of particles in drum mixers using DEM. Advanced Powder Technology, 27(2), 531-540. https://doi.org/10.1016/ 
j.apt.2016.01.016

Zhang, Z., Gui, N., Ge, L. and Li, Z. (2017). Numerical study of mixing of binary-size particles in rotating tumblers on the effects of end-walls and size ratios. Powder Technology, 314, 164-174. https:// doi.org/10.1016/j.powtec.2016.09.072

Zhang, Z., Wang, L., Wang, Y. and Chu, X. (2019). Discrete element simulation on mixing of granular materials in rotated container. Engineering Analysis with Boundary Elements, 106, 20-26. https:// doi.org/10.1016/j.enganabound.2019.04.034 